\title{
N990-13416
}

\section{Periodic-Disturbance Accommodating Control of the Space Station for Asymptotic Momentum Management *}

\author{
Wayne Warren ${ }^{\dagger}$ and Bong Wie \\ The University of Texas at Austin \\ Austin, Texas \\ David Geller ${ }^{\S}$ \\ NASA Johnson Space Center \\ Houston, Texas
}

\begin{abstract}
Periodic-disturbance accommodating control is investigated for asymptotic momentum management of control moment gyros used as primary actuating devices for the Space Station. The proposed controller utilizes the concepts of quaternion feedback control and periodic-disturbance accommodation to achieve oscillations about the constant torque equilibrium attitude, while minimizing the control effort required. Three-axis coupled equations of motion, written in terms of quaternions, are derived for roll/yaw controller design and stability analysis. The quaternion feedback controller designed using the linear-quadratic regulator synthesis technique is shown to be robust for a wide range of pitch angles. It is also shown that the proposed controller tunes the open-loop unstable vehicle to a stable oscillatory motion which minimizes the control effort needed for steady-state operations.
\end{abstract}

\section{Introduction}

The Space Station will employ CMGs (control moment gyros) as primary actuating devices during normal flight mode operation. Gravity-gradient torques will be used for CMG momentum management (unloading). The effect of a constant aerodynamic torque on a gravitationally stabilized spacecraft was first studied by Garber [1]. Such a torque produces a constant attitude angle for which aerody-

*'This work was supported by the NASA Johnson Space Center through the RICIS program of the University of Houston at Clear Lake.

${ }^{\dagger}$ Graduate Research Assistant, Member AIAA.

Assistant Professor, Dept. of Aerospace Engineering and Engineering Mechanics, Member AIAA.

$\$$ Aerospace Engineer, Mission Planning and Analysis Division. namic and gravitational torques are balanced. Garber [1] has shown that small roll/yaw librational motions are affected by large pitch angles. The aerodynamic disturbance torques acting on the Space Station are expected to have constant values plus periodic components caused mostly by the effects of solar panel rotations and Earth's diurnal bulge. As a result, attitude and CMG momentum oscillation about the torque equilibrium attitude will occur. A recent study [2] demonstrates the usefulness of the linear-quadratic-regulator synthesis technique and the concept of periodic-disturbance accommodation in minimizing attitude and/or CMG momentum oscillations as needed for mission requirements.

This paper is primarily concerned with attitude control and CMG periodic-disturbance rejection for large-angle pitch maneuvers of the Space Station. New results are presented expanding on the control scheme developed in [2]. Pitch-coupled roll/yaw equations of motion, first discussed in [1], and written in terms of Euler angles, are derived here in terms of quaternions. It is shown that these equations are well suited for use in designing a roll/yaw controller for large pitch motions of the Space Station. A simple concept of using quaternions for the control of spacecraft large-angle maneuvers has been developed in $[3,4]$. The concept is extended here to a more complicated case of controlling both the attitude and CMG momentum of the Space Station. Furthermore, this paper presents a new control concept of asymptotic momentum management of the CMGs, which tunes the open-loop unstable vehicle to a stable oscillatory notion during steadystate operations, while minimizing the control effort needed.

Figure 1 is a functional block diagram representation of a quaternion feedback control system proposed for the Space Station. The attitude determination system utilizes rate gyros and star trackers to compute inertial quaternions and the absolute angu- 
lar velocity of the Space Station. Relative quaternions with respect to a local reference frame are then computed for control purposes. The proposed attitude/momentum controller utilizes relative quaternions, body rates (or relative quaternion rates), and CMG momenta to generate proper control torque commands to the CMGs.

\section{Mathematical Models}

In this section, equations of motion for the Space Station in a circular orbit are derived in terms of quaternions. For simplicity, the Space Station is assumed to be a single rigid body. Emphasis is on the use of quaternions in the equations of motion and in feedback control. Quaternions define the rigid body attitude as an Euler-axis rotation. The vector part of the quaternions indicates the direction of the Euler axis. The scalar part of the quaternions is related to the rotation angle about the Euler axis. Detailed discussion of the kinematics associated with quaternions and Euler angles can be found in $[5,6]$.

The relationships between quaternions and Euler angles, for the pitch-yaw-roll body-axis rotation sequence used in this paper, are

$$
\left[\begin{array}{l}
q_{1} \\
q_{2} \\
q_{3} \\
q_{4}
\end{array}\right]=\left[\begin{array}{l}
C_{1} S_{2} S_{3}+S_{1} C_{2} C_{3} \\
C_{1} S_{2} C_{3}+S_{1} C_{2} S_{3} \\
C_{1} C_{2} S_{3}-S_{1} S_{2} C_{3} \\
C_{1} C_{2} C_{3}-S_{1} S_{2} S_{3}
\end{array}\right]
$$

where $C_{i} \triangleq \cos \left(\theta_{i} / 2\right), S_{i} \triangleq \sin \left(\theta_{i} / 2\right)$ for $i=1,2,3$, $\left(\theta_{1}, \theta_{2}, \theta_{3}\right)$ are the roll, pitch, and yaw Euler angles of the body axes with respect to the local vertical and local horizontal (LVLH) axes, which rotate with the orbital angular velocity; and $\left(q_{1}, q_{2}, q_{3}\right)$ are the vector parts of the quaternions which indicate the direction of the Euler axis while $q_{4}$ is the scalar part of the quaternions and is related to the angle of rotation about the Euler axis. Inverse relations may also be written as:

$$
\begin{aligned}
& \theta_{1}=\tan ^{-1}\left[\frac{2\left(q_{1} q_{4}-q_{2} q_{3}\right)}{1-2 q_{1}^{2}-2 q_{3}^{2}}\right] \\
& \theta_{2}=\tan ^{-1}\left[\frac{2\left(q_{2} q_{4}-q_{1} q_{3}\right)}{1-2 q_{2}^{2}-2 q_{3}^{2}}\right] \\
& \theta_{3}=\sin ^{-1}\left[2\left(q_{1} q_{2}+q_{3} q_{4}\right)\right] .
\end{aligned}
$$

The nonlinear equations of motion and attitude kinematics for the Space Station are as follows:
Space Station Dynamics:

$$
\begin{gathered}
{\left[\begin{array}{ccc}
I_{11} & I_{12} & I_{13} \\
I_{21} & I_{22} & I_{23} \\
I_{31} & I_{32} & I_{33}
\end{array}\right]\left[\begin{array}{l}
\dot{\omega}_{1} \\
\dot{\omega}_{2} \\
\dot{\omega}_{3}
\end{array}\right]=} \\
-\tilde{\omega}\left[\begin{array}{lll}
I_{11} & I_{12} & I_{13} \\
I_{21} & I_{22} & I_{23} \\
I_{31} & I_{32} & I_{33}
\end{array}\right]\left[\begin{array}{l}
\omega_{1} \\
\omega_{2} \\
\omega_{3}
\end{array}\right] \\
+3 n^{2} \tilde{\mathrm{c}}\left[\begin{array}{lll}
I_{11} & I_{12} & I_{13} \\
I_{21} & I_{22} & I_{23} \\
I_{31} & I_{32} & I_{33}
\end{array}\right]\left[\begin{array}{l}
c_{1} \\
c_{2} \\
c_{3}
\end{array}\right] \\
+\left[\begin{array}{l}
-u_{1}+w_{1} \\
-u_{2}+w_{2} \\
-u_{3}+w_{3}
\end{array}\right]
\end{gathered}
$$

where

$$
\begin{aligned}
& \tilde{\omega} \triangleq\left[\begin{array}{ccc}
0 & -\omega_{3} & \omega_{2} \\
\omega_{3} & 0 & -\omega_{1} \\
-\omega_{2} & \omega_{1} & 0
\end{array}\right] \\
& \tilde{\mathbf{c}} \triangleq\left[\begin{array}{ccc}
0 & -c_{3} & c_{2} \\
c_{3} & 0 & -c_{1} \\
-c_{2} & c_{1} & 0
\end{array}\right] \\
& c_{1} \triangleq 2\left(q_{1} q_{3}-q_{2} q_{4}\right) \\
& c_{2} \triangleq 2\left(q_{1} q_{4}+q_{2} q_{3}\right) \\
& c_{3} \triangleq 1-2 q_{1}^{2}-2 q_{2}^{2}
\end{aligned}
$$

Attitude Kinematics (with respect to LVLH):

$$
\left[\begin{array}{c}
\dot{q}_{1} \\
\dot{q}_{2} \\
\dot{q}_{3} \\
\dot{q}_{4}
\end{array}\right]=\frac{1}{2} \Omega\left[\begin{array}{l}
q_{1} \\
q_{2} \\
q_{3} \\
q_{4}
\end{array}\right]
$$

where

$$
\mathbf{\Omega} \triangleq\left[\begin{array}{cccc}
0 & \omega_{3} & -\omega_{2}+n & \omega_{1} \\
-\omega_{3} & 0 & \omega_{1} & \omega_{2}+n \\
\omega_{2}-n & -\omega_{1} & 0 & \omega_{3} \\
-\omega_{1} & -\omega_{2}-n & -\omega_{3} & 0
\end{array}\right]
$$

CMG Momentum:

$$
\left[\begin{array}{l}
\dot{h}_{1} \\
\dot{h}_{2} \\
\dot{h}_{3}
\end{array}\right]+\bar{\omega}\left[\begin{array}{l}
h_{1} \\
h_{2} \\
h_{3}
\end{array}\right]=\left[\begin{array}{l}
u_{1} \\
u_{2} \\
u_{3}
\end{array}\right]
$$

and $\left(\omega_{1}, \omega_{2}, \omega_{3}\right)$ are the body-axis components of the absolute angular velocity of the Space Station; $I_{i j}(i=j)$ are the moments of inertia; $I_{i j}(i \neq j)$ are the products of inertia; $\left(h_{1}, h_{2}, h_{3}\right)$ are the body-axis components of the CMG momentum; ( $u_{1}$, $\left.u_{2}, u_{3}\right)$ are the body-axis components of the control 
torque; $\left(w_{1}, w_{2}, w_{3}\right)$ are the body-axis components of the external disturbance torque; and $n$ is the orbital rate of $0.0011 \mathrm{rad} / \mathrm{sec}$.

When body and control axes are aligned with the principal axes of the Space Station $\left(I_{1} \triangleq I_{11}\right.$, $I_{2} \triangleq I_{22}, I_{3} \triangleq I_{33}$ ), Eqs. (3) become

$$
\begin{aligned}
& I_{1} \dot{\omega}_{1}-\left(I_{2}-I_{3}\right) \omega_{2} \omega_{3} \\
& +6 n^{2}\left(I_{2}-I_{3}\right)\left(q_{1} q_{4}+q_{2} q_{3}\right)\left(1-2 q_{1}^{2}-2 q_{2}^{2}\right) \\
& =-u_{1}+w_{1} \\
& I_{2} \dot{\omega}_{2}-\left(I_{3}-I_{1}\right) \omega_{1} \omega_{3} \\
& +6 n^{2}\left(I_{3}-I_{1}\right)\left(q_{1} q_{3}-q_{2} q_{4}\right)\left(1-2 q_{1}^{2}-2 q_{2}^{2}\right) \\
& =-u_{2}+w_{2} \\
& I_{3} \dot{\omega}_{3}-\left(I_{1}-I_{2}\right) \omega_{1} \omega_{2} \\
& +12 n^{2}\left(I_{1}-I_{2}\right)\left(q_{1} q_{3}-q_{2} q_{4}\right)\left(q_{1} q_{4}+q_{2} q_{3}\right) \\
& =-u_{3}+w_{3} .
\end{aligned}
$$

which can be found in [5]. In this paper, however, we present a new set of equations determined by linearizing the above equations for the case of large pitch angles with small roll/yaw attitude changes. In this case, Eqs. (4), (5), and (6) can be linearized with respect to $q_{1}$ and $q_{3}$ as follows:

Space Station Dynamics:

$$
\begin{aligned}
& I_{1} \dot{\omega}_{1}+n\left(I_{2}-I_{3}\right) \omega_{3}+6 n^{2}\left(I_{2}-I_{3}\right)\left(q_{4}-2 q_{4} q_{2}^{2}\right) q_{1} \\
& +6 n^{2}\left(I_{2}-I_{3}\right)\left(2 q_{2} q_{4}^{2}-q_{2}\right) q_{3}=-u_{1}+w_{1} \\
& I_{2} \dot{\omega}_{2}+6 n^{2}\left(I_{1}-I_{3}\right)\left(q_{2} q_{4}-2 q_{4} q_{2}^{3}\right) \\
& =-u_{2}+w_{2} \\
& I_{3} \dot{\omega}_{3}+n\left(I_{1}-I_{2}\right) \omega_{1}-12 n^{2}\left(I_{1}-I_{2}\right)\left(q_{2} q_{4}^{2}\right) q_{1} \\
& -12 n^{2}\left(I_{1}-I_{2}\right)\left(q_{4} q_{2}^{2}\right) q_{3}=-u_{3}+w_{9}
\end{aligned}
$$

Attitude Kinematics (with respect to LVLH):

$$
\begin{aligned}
& \dot{q}_{1}=\frac{q_{4}}{2} \omega_{1}+\frac{q_{2}}{2} \omega_{3}+n q_{3} \\
& \dot{q}_{2}=\frac{q_{4}}{2}\left(\omega_{2}+n\right) \\
& \dot{q}_{3}=-\frac{q_{2}}{2} \omega_{1}+\frac{q_{4}}{2} \omega_{3}-n q_{1} \\
& \dot{q}_{4}=-\frac{q_{2}}{2}\left(\omega_{2}+n\right)
\end{aligned}
$$

CMG Momentum:

$$
\begin{aligned}
\dot{h}_{1}-n h_{3} & =u_{1} \\
\dot{h}_{2} & =u_{2} \\
\dot{h}_{3}+n h_{1} & =u_{3} .
\end{aligned}
$$

For the case with large pitch angles and small roll/yaw attitude changes, the relationships between quaternions and Euler angles can be simplified by linearizing with respect to $\theta_{1}$ and $\theta_{3}$. Equations (1) then reduce to

$$
\begin{aligned}
q_{2} & =\sin \frac{\theta_{2}}{2} \\
q_{4} & =\cos \frac{\theta_{2}}{2} \\
{\left[\begin{array}{l}
q_{1} \\
q_{3}
\end{array}\right] } & =\frac{1}{2}\left[\begin{array}{rr}
q_{4} & q_{2} \\
-q_{2} & q_{4}
\end{array}\right]\left[\begin{array}{l}
\theta_{1} \\
\theta_{3}
\end{array}\right] .
\end{aligned}
$$

Inverse relations for Eqs. (10c) are written as

$$
\left[\begin{array}{l}
\theta_{1} \\
\theta_{3}
\end{array}\right]=2\left[\begin{array}{rr}
q_{4} & -q_{2} \\
q_{2} & q_{4}
\end{array}\right]\left[\begin{array}{l}
q_{1} \\
q_{3}
\end{array}\right]
$$

Equations (7) and (8) may be used to derive the Space Station equations of motion in terms of quaternions, written as follows:

$$
\begin{aligned}
& {\left[\begin{array}{rr}
I_{1} q_{4} & -I_{1} q_{2} \\
I_{3} q_{2} & I_{3} q_{4}
\end{array}\right]\left[\begin{array}{l}
\ddot{q}_{1} \\
\ddot{q}_{3}
\end{array}\right]} \\
& +n\left(I_{1}-I_{2}+I_{3}\right)\left[\begin{array}{rr}
-q_{2} & -q_{4} \\
q_{4} & -q_{2}
\end{array}\right]\left[\begin{array}{l}
\dot{q}_{1} \\
\dot{q}_{3}
\end{array}\right] \\
& +n^{2}\left[\begin{array}{cc}
\left(I_{2}-I_{3}\right) & 0 \\
0 & \left(I_{2}-I_{1}\right)
\end{array}\right] \\
& \quad \times\left[\begin{array}{cc}
\left(4 q_{4}-6 q_{4} q_{2}^{2}\right) & \left(6 q_{2} q_{4}^{2}-4 q_{2}\right) \\
\left(q_{2}+6 q_{2} q_{4}^{2}\right) & \left(q_{4}+6 q_{4} q_{2}^{2}\right)
\end{array}\right]\left[\begin{array}{c}
q_{1} \\
q_{3}
\end{array}\right] \\
& =\frac{1}{2}\left[\begin{array}{c}
-u_{1}+w_{1} \\
-u_{3}+w_{3}
\end{array}\right] \\
& I_{2}\left[q_{4} \ddot{q}_{2}-q_{2} \ddot{q}_{4}\right]+3 n^{2}\left(I_{3}-I_{1}\right)\left(2 q_{4} q_{2}^{3}-q_{4} q_{2}\right) \\
& =\frac{1}{2}\left(-u_{2}+w_{2}\right) .
\end{aligned}
$$

The quaternion relations of Eqs. (10) may be used to transform Eqs. (12) to the following known form $[1,6]$ involving only Euler angles:

$$
\begin{aligned}
& I_{1} \ddot{\theta}_{1}+n^{2}\left(I_{2}-I_{3}\right)\left(1+3 \cos ^{2} \theta_{2}\right) \theta_{1} \\
& -n\left(I_{1}-I_{2}+I_{3}\right) \dot{\theta}_{3}+3 n^{2}\left(I_{2}-I_{3}\right)\left(\sin \theta_{2} \cos \theta_{2}\right) \theta_{3} \\
& =-u_{1}+w_{1} \\
& I_{2} \ddot{\theta}_{2}+3 n^{2}\left(I_{1}-I_{3}\right) \sin \theta_{2} \cos \theta_{2} \\
& =-u_{2}+w_{2} \\
& I_{3} \ddot{\theta}_{3}+n^{2}\left(I_{2}-I_{1}\right)\left(1+3 \sin ^{2} \theta_{2}\right) \theta_{3} \\
& +n\left(I_{1}-I_{2}+I_{3}\right) \dot{\theta}_{1}+3 n^{2}\left(I_{2}-I_{1}\right)\left(\sin \theta_{2} \cos \theta_{2}\right) \theta_{1} \\
& =-u_{3}+w_{3} .
\end{aligned}
$$

A final linearization with respect to small pitch motions leads to the following well-known equations of motion:

Space Station Dynamics:

$$
I_{1} \ddot{\theta}_{1}+4 n^{2}\left(I_{2}-I_{3}\right) \theta_{1}
$$




$$
\begin{aligned}
& -n\left(I_{1}-I_{2}+I_{3}\right) \dot{\theta}_{3}=-u_{1}+w_{1} \\
& I_{2} \ddot{\theta}_{2}+3 n^{2}\left(I_{1}-I_{3}\right) \theta_{2}=-u_{2}+w_{2} \\
& I_{3} \ddot{\theta}_{3}+n^{2}\left(I_{2}-I_{1}\right) \theta_{3} \\
& +n\left(I_{1}-I_{2}+I_{3}\right) \dot{\theta}_{1}=-u_{3}+w_{3}
\end{aligned}
$$

Attitude Kinematics (with respect to LVLH):

$$
\begin{aligned}
\dot{\theta}_{1}-n \theta_{3} & =\omega_{1} \\
\dot{\theta}_{2}-n & =\omega_{2} \\
\dot{\theta}_{3}+n \theta_{1} & =\omega_{3} .
\end{aligned}
$$

These linearized equations are used in [2] for the case of small roll, pitch, and yaw attitude changes. In this paper, emphasis is on the use of Eqs. (7) for the momentum/attitude control of the Space Station having small roll/yaw attitude changes but large-angle pitch motions.

Inertia values for the Phase 1 Space Station, as well as assembly flight 3 , are listed in Table 1. Table 2 includes expected aerodynamic disturbances which are modeled as a bias plus periodic terms in the body-fixed control axes:

$$
\begin{aligned}
\mathrm{w}(t)=\mathrm{Bias} & +A_{n} \sin \left(n t+\phi_{1}\right) \\
& +A_{2 n} \sin \left(2 n t+\phi_{2}\right) \\
& +A_{3 n} \sin \left(3 n t+\phi_{3}\right) \\
& +A_{4 n} \sin \left(4 n t+\phi_{4}\right)
\end{aligned}
$$

The disturbance torque acting in each axis is determined from data generated at NASA Johnson Space Center by a nonlinear simulation program. The program simulates translational and rotational motions of the Space Station in orbit about an oblate Earth. It includes rotating solar panels, time-varying surface areas, and time-varying center-of-pressure locations. A Jacchia-Lineberry atmospheric model is used to compute density variations. The dominant aerodynamic torque frequencies at $n$ and $2 n$ are caused by Earth's diurnal bulge and solar panel rotation effects, respectively. Actual magnitudes and phases of these disturbance torques are assumed unknown for control design.

\section{Control Issues}

Before presenting the pitch and roll/yaw controller designs, it is important to clarify some issues related to the effects of large pitch motions and inertia value uncertainties on the stability of the controlled Space Station. A characteristic of momentum/attitude control using gravity-gradient torque is that pitch, roll, and yaw responses will settle down to, or oscillate about, a constant torque equilibrium attitude (TEA). Primary factors involved in determining the constant pitch TEA are the magnitude of the bias in the disturbance torque and the numerical difference between roll and yaw moments of inertia. This can be seen by studying the steady-state form of Eq. (14b):

$$
\overline{\theta_{2}}=\frac{\overline{\mathbf{w}}}{3 n^{2}\left(I_{1}-I_{3}\right)}
$$

where $\overline{\theta_{2}}$ is the pitch TEA angle and $\bar{w}$ is the bias of the pitch disturbance torque. Pitch gravity-gradient torque is largest when the pitch attitude is 45 degrees. This is predicted in Eq. (13b) where, at the steady-state,

$$
\sin \left(2 \overline{\theta_{2}}\right)=\frac{2 \overline{\mathrm{w}}}{3 n^{2}\left(I_{1}-I_{3}\right)} .
$$

It may be necessary to consider this worst-case pitch TEA in control system design. After switching to CMG mode from some other modes (e.g., reboost mode utilizing reaction jets), the Space Station must be able to achieve TEA in each axis without CMG momentum or commanded torque saturations.

The large motions possible in the pitch axis emphasize the importance of using Eqs. (7), (12), or (13) in designing the control system. These equations show the dependence of roll/yaw dynamics on pitch attitude. In fact, a roll/yaw closed-loop system designed for small pitch angles may become unstable at large pitch angles; therefore, roll/yaw closed-loop stability must be checked at various pitch TEA values.

Other factors affecting closed-loop stability are uncertainties and variations in moments and products of inertia. In particular, the magnitude of the pitch gravity-gradient torque depends on the difference between the roll and yaw moments of inertia. If these inertias are nearly equal (e.g., see Table 1, assembly flight 3 data), pitch gravity-gradient torque is very small and the pitch TEA (if it exists) is large. In addition, very small uncertainties in the moments of inertia can cause the system to become unstable. A discussion of the importance of checking closedloop system robustness with respect to inertia uncertainties, by varying inertia values in an appropriate "direction", is included in the appendix of this paper.

Under normal operating conditions, the Space Station will have rotating solar arrays. This causes time-varying (sinusoidal) roll and yaw moments of inertia, and consequently, a similarly time-varying gravity-gradient torque in the pitch axis as shown 
in Fig. 2. If the bias value of $I_{1}(t)-I_{3}(t)$ is such that the pitch gravity-gradient torque never changes sign, the system will remain stable. If the gravitygradient torque does switch signs, the system may become unstable, depending upon how long the sign of the torque is changed. For sufficiently short periods of this opposite torque, the system will be stable with large, bounded responses about the TEA. If gravity-gradient torque is to ultimately be used in momentum/attitude control, the above factors will be useful in the design of the inertia configuration. Consideration should be given to defining a boundary near the point or condition corresponding to zero pitch-axis gravity-gradient torque, defined by $I_{1}(t)-I_{3}(t)=0$. This is illustrated in Fig. 2 . The boundary may be thought of as the minimum gravity-gradient torque allowed in the pitch axis for which closed-loop stability, with respect to inertia uncertainties, is maintained.

In the next sections, the pitch and roll/yaw controller designs are presented along with time simulations of the closed-loop system. In this paper, we expand on the previous study [2] by developing a periodic-disturbance accommodating controller which achieves asymptotic momentum management of the CMGs in all three axes. The Phase 1 inertia configuration listed in Table 1 is used. Effects of products of inertia are assumed negligible. The corresponding time-varying gravity-gradient torque in the pitch axis does not change sign; however, the time simulations presented here use the assumption that inertia values remain constant while periodic terms in the aerodynamic torque include solar panel rotation effects. Large pitch TEA responses are produced by introducing an appropriately large bias in the pitch-axis disturbance torque.

\section{Pitch Control}

In this section, a pitch-axis controller is developed for attitude and CMG momentum control. It is shown in [2] that disturbance rejection filters can be used to reject either attitude or CMG momentum oscillations occurring at the frequencies present in the disturbance torques. Since asymptotic momentum management of the CMGs in all three axes is of primary interest in this paper, the disturbance rejection filters for the pitch axis have the following forms:

$$
\begin{aligned}
& \ddot{\alpha}_{2}+(n)^{2} \alpha_{2}=h_{2} \\
& \ddot{\beta}_{2}+(2 n)^{2} \beta_{2}=h_{2}
\end{aligned}
$$

$$
\begin{aligned}
& \ddot{\gamma}_{2}+(3 n)^{2} \gamma_{2}=h_{2} \\
& \ddot{\eta}_{2}+(4 n)^{2} \eta_{2}=h_{2}
\end{aligned}
$$

where initial conditions for the filter states can be arbitrarily selected (usually zero initial conditions). Use of filters at frequencies $n, 2 n, 3 n$, and $4 n$ is indicated by aerodynamic torque data generated by a nonlinear simulation program written for the Space Station. The pitch-axis control logic is given by a single control input involving twelve states:

$$
u_{2}=\mathbf{K}_{2} \mathbf{x}_{2}
$$

where

$$
\begin{aligned}
& \mathbf{K}_{2} \triangleq \text { a } 1 \times 12 \text { gain matrix } \\
& \mathbf{x}_{2} \triangleq\left[\begin{array}{ccccccc}
q_{2} & \dot{q}_{2} & h_{2} & \int h_{2} & \alpha_{2} & \dot{\alpha}_{2} & \\
\beta_{2} & \dot{\beta}_{2} & \gamma_{2} & \dot{\gamma}_{2} & \eta_{2} & \dot{\eta}_{2} & ]^{T}
\end{array}\right.
\end{aligned}
$$

The control task is to find proper gains for this twelve-state feedback controller.

In order to use linear control design methodologies, Eq. (7b) must be linearized for small pitch motion. This results in Eq. (14b), which is used as the basis for pitch control analysis and design. Various techniques may be used in selecting the twelve gains of Eq. (16). These include linear-quadraticregulator (LQR) synthesis [7] and direct assignment of closed-loop eigenvalues using a pole-placement technique. Several iterations of any method may be required to achieve satisfactory closed-loop performance and robustness. Note that gains resulting from Eqs. (14b) are for the use of $\theta_{2}$ in state feedback. In order to accommodate $q_{2}$ for use in feedback, the gains corresponding to states $q_{2}$ and $\dot{q}_{2}$ are doubled since the approximation used for $q_{2}$ is $\theta_{2} / 2$. New gains do not need to be computed for the pitch controller in the case of large pitch motion. It is mostly roll/yaw destabilization at large pitch angles which forces pitch-axis instability.

The open-loop pitch axis of the Phase 1 inertia configuration is unstable, with poles at $\mathrm{s}= \pm 1.5 n$, 0,0 , and filter poles at $\mathrm{s}= \pm \mathrm{j} n, \pm \mathrm{j} 2 n, \pm \mathrm{j} 3 n, \pm \mathrm{j} 4 n$. One pole at $\mathrm{s}=0$ comes from the integral feedback of $h_{2}$. After itcrative use of an LQR synthesis code, available in CTRL-C software, a set of closed-loop eigenvalues have been selected and are listed in Table 3 . The corresponding gain set is given in Table 4. Closed-loop pitch responses of $\mathrm{Eq}$. (6), for a pitchaxis maneuver of -30 degrees (caused by a large pitch-axis torque bias), can be seen in Figs. 4, 5 , and 6 . Comments on the responses are reserved until after the presentation of the roll/yaw controller design. 


\section{Roll/Yaw Control}

The roll/yaw controller has a structure similar to that of the pitch controller. By examining the openloop transfer function matrix from control inputs to roll/yaw attitudes and CMG momentum, it is shown in [2] that a periodic disturbance at the orbital rate can be rejected in the yaw attitude but not in the roll attitude. The analysis is accomplished using Eqs. (9) and (14), which assume small motions in all axes. In an effort to determine if it is possible to have periodic-disturbance rejection in both roll and yaw attitudes for this different case involving large pitch motions, a similar analysis is considered here using the pitch-coupled roll/yaw dynamics described by Eqs. (13).

By combining Eqs. (9) and (13), the transfer function matrix from $\left(u_{1}, u_{3}\right)$ to $\left(\theta_{1}, \theta_{3}\right)$ can be written as

$$
\left[\begin{array}{l}
\theta_{1}(s) \\
\theta_{3}(s)
\end{array}\right]=\frac{1}{\Delta}\left[\begin{array}{ll}
G_{11}(s) & G_{13}(s) \\
G_{31}(s) & G_{33}(s)
\end{array}\right]\left[\begin{array}{l}
u_{1}(s) \\
u_{3}(s)
\end{array}\right]
$$

where

$$
\begin{aligned}
G_{11}(s)= & -\left[I_{3} s^{2}+\left(1+3 s_{2}^{2}\right) n^{2}\left(I_{2}-I_{1}\right)\right] \\
& \times\left[s^{2}+n^{2}\right] \\
G_{13}(s)= & -\left[n\left(I_{1}-I_{2}+I_{3}\right) s\right. \\
& \left.-3 n^{2}\left(I_{2}-I_{3}\right) s_{2} c_{2}\right]\left[s^{2}+n^{2}\right] \\
G_{31}(s)= & {\left[n\left(I_{1}-I_{2}+I_{3}\right) s\right.} \\
& \left.+3 n^{2}\left(I_{2}-I_{1}\right) s_{2} c_{2}\right]\left[s^{2}+n^{2}\right] \\
G_{33}(s)= & -\left[I_{1} s^{2}+\left(1+3 c_{2}^{2}\right) n^{2}\left(I_{2}-I_{3}\right)\right] \\
& \times\left[s^{2}+n^{2}\right] \\
\Delta= & I_{1} I_{3}\left(s^{2}+n^{2}\right)\left\{s^{4}+n^{2}\left[1+3 k_{1}+k_{1} k_{3}\right.\right. \\
& \left.+3\left(k_{3}-k_{1}\right) s_{2}^{2}\right] s^{2} \\
& \left.+\left[3 n^{3}\left(k_{3}-k_{1}\right) s_{2} c_{2}\right] s+4 n^{4} k_{1} k_{3}\right\}
\end{aligned}
$$

and $s_{2}=\sin \theta_{2}, c_{2}=\cos \theta_{2}, k_{1}=\left(I_{2}-I_{3}\right) / I_{1}$, and $k_{3}=\left(I_{2}-I_{1}\right) / I_{3}$. Transmission zeros at $\pm j n$ appear in the transfer function matrix. It would seem that periodic disturbances of frequency $n$ cannot be rejected in either the roll or yaw attitudes; however, for CMG momentum and control torque relations defined by

$$
\begin{aligned}
& \dot{h}_{1}=u_{1} \\
& \dot{h}_{3}=u_{3}
\end{aligned}
$$

and with appropriate alterations of Eqs. (13), the transfer function matrix from $\left(u_{1}, u_{3}\right)$ to $\left(\theta_{1}, \theta_{3}\right)$ can be written as

$$
\left[\begin{array}{l}
\theta_{1}(s) \\
\theta_{3}(s)
\end{array}\right]=\frac{1}{\Delta}\left[\begin{array}{ll}
G_{11}(s) & G_{13}(s) \\
G_{31}(s) & G_{33}(s)
\end{array}\right]\left[\begin{array}{l}
u_{1}(s) \\
u_{3}(s)
\end{array}\right]
$$

where

$$
\begin{aligned}
G_{11}= & (s)\left\{-I_{3} s^{3}+n^{2}\left[3\left(I_{1}-I_{2}\right) s_{2}^{2}-I_{3}\right] s\right. \\
& \left.+3 n^{3}\left(I_{2}-I_{3}\right) s_{2} c_{2}\right\} \\
G_{13}= & (n s)\left\{\left(I_{2}-I_{1}\right) s^{2}+\left[3 n\left(I_{2}-I_{3}\right) s_{2} c_{2}\right] s\right. \\
& \left.+\left(1+3 s_{2}^{2}\right) n^{2}\left(I_{2}-I_{1}\right)\right\} \\
G_{31}= & -(n s)\left\{\left(I_{2}-I_{3}\right) s^{2}+\left(1+3 c_{2}^{2}\right) n^{2}\left(I_{2}-I_{3}\right)\right\} \\
G_{33}= & -\left\{I_{1} s^{4}+n^{2}\left[3\left(I_{2}-I_{3}\right) c_{2}^{2}+I_{1}\right] s\right. \\
& \left.+3 n^{2}\left(I_{2}-I_{1}\right) s_{2} c_{2}\right\} \\
\Delta= & I_{1} I_{3}\left(s^{2}\right)\left\{s^{4}+n^{2}\left[1+3 k_{1}+k_{1} k_{3}\right.\right. \\
& \left.+3\left(k_{3}-k_{1}\right) s_{2}^{2}\right] s^{2} \\
& \left.+\left[3 n^{3}\left(k_{3}-k_{1}\right) s_{2} c_{2}\right] s+4 n^{4} k_{1} k_{3}\right\} .
\end{aligned}
$$

Transmission zeros are not apparent in these expressions. A numerical analysis reveals, however, that there are transmission zeros at $\pm j n$ for the transfer functions from $u_{1}$ and $u_{3}$ to roll attitude, while yaw attitude has no troublesome zeros. These results show that, even for the case of pitch-coupled roll/yaw dynamics, there is an inability to reject roll attitude oscillations occurring at the orbital rate. Hence, in this paper, periodic-disturbance rejection for CMG momentum in both the roll and yaw axes is considered. That is, a periodic control of the Space Station for asymptotic momentum management of the CMGs in all three axes is of primary interest here.

Periodic-disturbance rejection filters for the roll/yaw axes can be represented as:

$$
\begin{aligned}
& \ddot{\alpha}_{1}+(n)^{2} \alpha_{1}=h_{1} \\
& \ddot{\beta}_{1}+(2 n)^{2} \beta_{1}=h_{1} \\
& \ddot{\gamma}_{1}+(3 n)^{2} \gamma_{1}=h_{1} \\
& \ddot{\eta}_{1}+(4 n)^{2} \eta_{1}=h_{1} \\
& \ddot{\alpha}_{3}+(n)^{2} \alpha_{3}=h_{3} \\
& \ddot{\beta}_{3}+(2 n)^{2} \beta_{3}=h_{3} \\
& \ddot{\gamma}_{3}+(3 n)^{2} \gamma_{3}=h_{3} \\
& \ddot{\eta}_{3}+(4 n)^{2} \eta_{3}=h_{3} .
\end{aligned}
$$

The roll/yaw control logic involving two control inputs and twenty-four states is expressed as

$$
\left[\begin{array}{l}
u_{1} \\
u_{3}
\end{array}\right]=\mathrm{K}\left[\begin{array}{l}
\mathbf{x}_{1} \\
\mathbf{x}_{3}
\end{array}\right]
$$

where

$\mathrm{K} \triangleq$ a $2 \times 24$ gain matrix

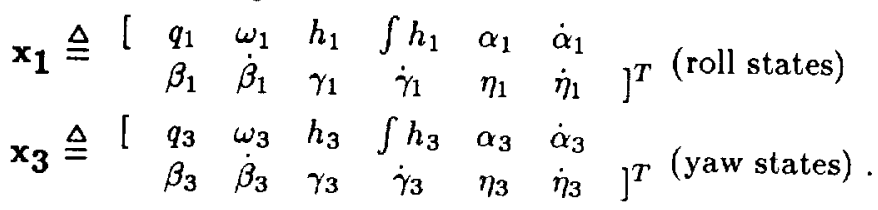


The pitch-coupled roll/yaw equations described by Eqs. (7) are used as the basis for the roll/yaw controller design. It is evident in the pitch-coupled equations that roll/yaw dynamics are dependent upon pitch attitude. Consideration must be given to the pitch-axis TEA when designing the roll/yaw controller gains. Equations (7) (or Eqs. (12) or (13)) are especially useful for this purpose when $q_{2}$ and $q_{4}$ in the equations are assigned their respective values corresponding to the expected pitch TEA (see Eqs. (10a) and (10b)). As a result, Eqs. (7a) and (7c) become linear and any linear control design methodologies may be used to design the roll/yaw controller gains.

For spacecraft operating under the influence of gravity-gradient torques, it is interesting to examine the changes in roll/yaw open-loop eigenvalues that occur as the pitch bias changes. This was first studied by Garber [1]. A root locus of open-loop eigenvalues versus pitch angle, for the Phase 1 inertia configuration, is shown in Fig. 3. It can be seen that the open-loop roll/yaw dynamics are not very sensitive to pitch attitude. The Space Station is unstable with poles at $\mathrm{s}= \pm 1.05 n \pm \mathrm{j} 0.7 n, 0,0$, $\pm \mathrm{j} n$, and filter poles at $\mathrm{s}= \pm \mathrm{j} n, \pm \mathrm{j} n, \pm \mathrm{j} 2 n, \pm \mathrm{j} 2 n$, $\pm \mathrm{j} 3 n, \pm \mathrm{j} 3 n, \pm \mathrm{j} 4 n, \pm \mathrm{j} 4 n$ (for $\theta_{2}=0^{\circ}$ where $q_{2}=0$ and $q_{4}=1$ ). The double pole at $\mathrm{s}=0$ occur because of the integral feedback of $h_{1}$ and $h_{3}$.

After iterative use of an LQR synthesis code, closed-loop eigenvalues have been selected and are listed in Table. 3. A gain set for Eqs. (17), corresponding to a pitch TEA of $0^{\circ}$, is listed in Table. 4. For these gains, the closed-loop roll/yaw axes are stable for pitch angles ranging from $-21^{\circ}$ to $+23^{\circ}$. Since a simulation of the large-angle pitch maneuver needed to reach a pitch TEA of $-30^{\circ}$ is desired, a different gain set is used for the simulations presented in this paper. For these gains, the closedloop roll/yaw axes are stable for pitch angles ranging from $-48^{\circ}$ to $+3^{\circ}$. Closed-loop roll/yaw responses of Eqs. (6), for a pitch-axis maneuver of -30 degrees, can be seen in Figs. 4, 5, and 6. The overal! closed-loop system has a $10 \mathrm{~dB}$ gain margin and a phase margin of $60^{\circ}$ in each control loop.

\section{Discussion of Simulation Results}

Closed-loop responses for a simulation of the nonlinear dynamics described by Eqs. (6) are shown in Figs. 4, 5, and, 6. Quantities plotted include quaternions, CMG momenta, and control torques. Initial conditions corresponding to $\theta_{1}(0)=\theta_{2}(0)=\theta_{3}(0)=$
1 degree and $\dot{\theta}_{1}(0)=\dot{\theta}_{2}(0)=\dot{\theta}_{3}(0)=0.001 \mathrm{deg} / \mathrm{sec}$ are assumed. Allowable limits on CMG momentum and commanded torque are assumed to be 30,000 $\mathrm{ft}-\mathrm{lb}-\mathrm{sec}$ and $150 \mathrm{ft}-\mathrm{lb}$, respectively.

In the roll axis, quaternion $q_{1}$ oscillates $\left( \pm 0.7^{\circ}\right)$ about a roll 'JEA of $-0.003\left(\theta_{1}=-0.5^{\circ}\right)$. Roll CMG momentum $h_{1}$ is the input to the roll-axis disturbance rejection filter and settles down to zero after reaching a maximum value near $8000 \mathrm{ft}$-lb-sec. Control torque $u_{1}$ is zero at the steady-state and has a peak value near $23 \mathrm{ft}-\mathrm{lb}$. In the pitch axis, quaternion $q_{2}$ oscillates $\left( \pm 4.3^{\circ}\right)$ about a pitch TEA of $-0.257\left(\theta_{2}=-30^{\circ}\right)$. 'The large-angle mancuver causes the pitch CNG momentum $h_{2}$ (disturbance filter input) to become quite large at nearly 18,000 $\mathrm{ft}$-lb-sec, before settling to zero. Control torque $u_{2}$ is zero at the steady-state with a maximum value near $30 \mathrm{ft}$-lb. In the yaw axis, quatemion $q_{3}$ oscillates $\left( \pm 1.2^{\circ}\right)$ about a yaw TEA of $-0.013\left(\theta_{3}=-1.5^{\circ}\right)$. Yaw CMG moment um $h_{3}$ (disturbance filter input) settles down to zero after reaching a maximum value close to $600 \mathrm{ft}-\mathrm{lb}$-sec. Control torque $u_{3}$ is zero at the steady-state, and reaches a maximum value near $12 \mathrm{ft}-\mathrm{lb}$.

The simulations show that the proposed control scheme tunes the open-loop unstable Space Station to a stable, oscillatory motion which minimizes control effort during steady-state operations. For the assumcd disturbance torque models (with unknown magnitudes and phases), the stabilized Space Station needs no control torque at. steadystate conditions, Analysis shows, however, that small-amplitude periodic components of frequencies $5 n$ and $6 n$ are present in $u_{1}$ and $u_{3}$ at the steadystate. These small residual components are caused by the compling between the pitch and roll/yaw axes, and become particularly noticeable for large pitch biases.

There is an interesting feature of the quaternion feedback scheme which is not apparent from the simulation responses. If rejection of pitch and yaw attitude oscillations is desired, it seems natural to use $q_{2}$ and $q_{3}$ as inputs to the respective disturbance rejection filters. Fven though $q_{2}$ and $q_{3}$ will become constant at the steady-state, all of the Euler angles will oscillate, $A$ study of Eqs. (2) (or Eqs. (11)) reveals why. By assigning constant values to $q_{2}, q_{3}$, and $q_{4}$ in Eqs. (2) (or Eqs. (11)), it can be seen that $\theta_{1}, \theta_{2}$, and $\theta_{3}$ are all functions of $q_{1}$, which oscillates. The same may be said for $q_{1}, q_{2}, q_{3}$, and $q_{4}$ if Euler angle feedback is used. For oscillations of $\theta_{1}$ and constant values for $\theta_{2}$ and $\theta_{3}$, Eqs. (1) show that all quaternions are functions of $\theta_{1}$ and will therefore oscillate. In cither case, however, these oscillations 
are small. The important point is that the elimination of pitch or yaw oscillations, if needed, may be accomplished by using Euler angles $\theta_{2}$ and $\theta_{3}$ as disturbance rejection filter inputs (with appropriate gain changes).

A check of closed-loop robustness with respect to inertia uncertainties emphasizes an important issue associated with the Phase 1 inertia configuration. By selecting specific "directions" in which to vary the three moments of inertia $\left(I_{1}, I_{2}, I_{3}\right)$, the closedloop system can be shown to be unstable for as little as $-7 \%$ uncertainty in $I_{3}$ with $+8 \%$ uncertainty in $I_{1}$. For these inertia variations, the pitch-axis gravity-gradient torque disappears $\left(I_{1}-I_{3}=0\right)$ and closed-loop pitch dynamics become unstable. The limitations shown in this example (and several others involving even smaller inertia uncertainties), are not related to the selection of control logic but are physical limitations inherent to the inertia configuration of the Phase 1 Space Station. A description of the inertia variation "directions" used above is presented in the appendix of this paper.

\section{Conclusions}

In this paper asymptotic momentum management of control moment gyros of the Space Station has been investigated. It was shown that the proposed controller tunes the Space Station, which has a gravitationally "unstable" inertia configuration, to a stable, oscillatory motion which minimizes the control effort needed at the steady-state. By utilizing the concepts of quaternion feedback control and periodic-disturbance rejection filters, the proposed controller provides robust control of the Space Station for large-angle pitch motions. The pitch-coupled roll/yaw equations of motion derived in this paper were shown to be particularly useful in roll/yaw controller design and stability analysis.

\section{Appendix: Inertia Variation Directions}

It is a common practice in control design to satisfy time and frequency-domain requirements first, then check for closed-loop robustness. For spacecraft, inertial properties may be very sensitive parameters in the closed-loop system. It seems reasonable to increase and decrease all inertias by the same percentage, thus checking controller effectiveness for a proportionally heavier and lighter spacecraft. This procedure may not indicate the true inertia sensi- tivity. It is important to consider the magnitude and direction of the variation for each inertia value. Since the inertia matrix may be transformed to three principal moments of inertia by aligning the body and principal axes, suggestions for variations in the moments of inertia for the roll $\left(I_{1}\right)$, pitch $\left(I_{2}\right)$, and yaw $\left(I_{3}\right)$ axes are presented here.

Three important relationships may be derived from the definitions for the moments of inertia. These relationships are as follows:

$$
\begin{aligned}
& I_{1}+I_{2}>I_{3} \\
& I_{1}+I_{3}>I_{2} \\
& I_{2}+I_{3}>I_{1} .
\end{aligned}
$$

Together, these relations define the physically possible inertia configurations. A control designer may unknowingly use inertia variations which result in inertia values that violate these constraints. Stability of the closed-loop system will be tested for a physically impossible inertia configuration. The important point is not the fictitious inertias but whether or not the control designer can redirect this extraneous stability margin to encompass more of the region of physically possible inertia values.

When gravity-gradient torque is used in the control of a rotating spacecraft, additional inertia constraints are introduced. The control scheme presented in this paper is a good example. Equations (14) show that roll-axis gravity-gradient and gyroscopic coupling torques are zero when $I_{2}=I_{3}$, pitch-axis gravity-gradient torque is zero when $I_{1}=$ $I_{3}$, and the yaw-axis gyroscopic coupling torque is zero when $I_{1}=I_{2}$

$A$ useful aid for visualizing the relationship between inertia constraints and inertia variations is now presented. Figure A.1 shows a threedimensional, cubic figure defined in three "inertial" directions. The inertia constraint relations may now be visualized as planes in this "inertial" space. The planes $I_{1}+I_{2}=I_{3}, I_{1}+I_{3}=I_{2}$, and $I_{2}+I_{3}=I_{1}$ are labeled in Fig. A.1, and represent the physical boundaries of inertia values. The area inside the three intersecting boundaries represents all physically possible inertia configurations. A representation of the cut-away portion of the "inertia cube" is shown in Fig. A.2. Planes defining the physical and system torque boundaries are labeled. Figures A.1 and $A .2$ provide a three-dimensional representation of the information presented in the $k_{3}$ versus $k_{1}$ inertia ratio plots in [5-7]. It may be convenient to normalize the moments of inertia being studied by $\sqrt{I_{1}^{2}+I_{2}^{2}+I_{3}^{2}}$ in order to locate the position of the nominal configuration within a "unit inertia cube". 
The relative positioning of the nominal inertia configuration from the constraint boundaries can then be easily determined.

Since the shortest distance from a point to a plane is in a direction perpendicular to that plane, it seems logical to check inertia variations in directions perpendicular to the inertia boundaries. In this way, the minimum variation necessary to reach a physical boundary can be found while checking the closedloop stability of the system in question. For rotating spacecraft with gravity-gradient control systems, inertia variations perpendicular to the planes $I_{1}=I_{2}$, $I_{1}=I_{3}$, and $I_{2}=I_{3}$ are needed. It may be seen in Fig. A.2 that these planes intersect inside the region of physically possible inertia values, and partition the region into several sections. It should be a control designers' goal to include the area within the physical boundaries inside a "control surface" which contains all of the inertia values for which the closed-loop system is stable.

\section{References}

1. Garber, T.B., "Influence of Constant Disturbing Torque on the Motion of Gravity-Gradient Stabilized Satellites," AIAA Journal, Vol.1, No.4, April 1963, pp. 968-969.

2. Wie, B., Byun, K.W., Warren, W., Geller, D., Long, D., and Sunkel, J., "A New Approach to Attitude/Momentum Control of the Space Station," AIAA Paper No.88-4132 (to appear in the Journal of Guidance, Control, and Dynamics).

3. Wie, B., and Barba, P.M., "Quaternion Feedback for Spacecraft Large-Angle Maneuvers," Journal of Guidance, Control, and Dynamics, Vol.8, No.3, May-June 1985, pp. 360-365.

4. Wie, B., Weiss, H., and Arapostathis, A., "Quaternion Feedback Regulator for Spacecraft Eigenaxis Rotations," AIAA Paper No.88-4117 (to appear in the Journal of Guidance, Control, and Dynamics).

5. Kane, T.R., Likins, P.W., and Levinson, D.A., Spacecraft Dynamics, McGraw-Hill, 1983, pp. 12-16, 23-25, 58-62.

6. Hughes, P.C., Spacecraft Attitude Dynamics, John-Wiley\&Sons, 1986, Chapters 2 and 9.
7. Bryson, A.E., "Control of Spacecraft and Aircraft," Course Notes, Stanford University (to be published as a text).

Table 1. Space station inertia configurations

\begin{tabular}{crr}
\hline $\left.\begin{array}{c}\text { Inertia } \\
(\text { slug-ft }\end{array}{ }^{2}\right)$ & $\begin{array}{r}\text { Assembly } \\
\text { Flight\#3 }\end{array}$ & Phase 1 \\
\hline$I_{11}$ & $23.22 \mathrm{E} 6$ & $50.28 \mathrm{E} 6$ \\
$I_{22}$ & $1.30 \mathrm{E} 6$ & $10.80 \mathrm{E} 6$ \\
$I_{33}$ & $23.23 \mathrm{E} 6$ & $58.57 \mathrm{E} 6$ \\
$I_{12}$ & $-0.023 \mathrm{E} 6$ & $-0.39 \mathrm{E} 6$ \\
$I_{13}$ & $0.477 \mathrm{E} 6$ & $-0.24 \mathrm{E} 6$ \\
$I_{23}$ & $-0.011 \mathrm{E} 6$ & $0.16 \mathrm{E} 6$ \\
\hline
\end{tabular}

Table 2. Phase 1 aerodynamic torque models (in units of ft-lbs)

\begin{tabular}{cc}
\hline $\mathrm{w}_{1}$ & $1+\sin (n \mathrm{t})+0.5 \sin (2 n \mathrm{t})$ \\
& $+03 \sin (3 n \mathrm{t})+0.5 \sin (4 n \mathrm{t})$ \\
$\mathrm{w}_{2}$ & $13^{*}+1.2 \sin (n \mathrm{t})+3.5 \sin (2 n \mathrm{t})$ \\
& $+0.3 \sin (3 n \mathrm{t})+0.5 \sin (4 n \mathrm{t})$ \\
$\mathrm{w}_{3}$ & $1+\sin (n \mathrm{t})+0.5 \sin (2 n \mathrm{t})$ \\
& $+0.3 \sin (3 n \mathrm{t})+0.5 \sin (4 n \mathrm{t})$
\end{tabular}

* nominal pitch bias torque is 4 but 13 is used to produce a large pitch TEA 
Table 3. Phase 1 closed-loop eigenvalues

(in units of orbital rate $-0.0011 \mathrm{rad} / \mathrm{sec}$ )

\begin{tabular}{l|cc|cc}
\hline & \multicolumn{2}{|c|}{ Momentum/Attitude } & \multicolumn{2}{c}{ Disturbance Filters } \\
\hline Pitch & $-1.0,-1.5$ & $-1.5 \pm \mathrm{j} 1.5$ & $-0.3 \pm \mathrm{j} 1.0$ & $-0.3 \pm \mathrm{j} 2.0$ \\
& & & $-0.3 \pm \mathrm{j} 3.0$ & $-0.3 \pm \mathrm{j} 4.0$ \\
\hline Roll/Yaw & $-0.23,-0.71$ & $-0.53 \pm \mathrm{j} 1.54$ & $-0.14 \pm \mathrm{j} 0.99$ & $-1.13 \pm \mathrm{j} 0.75$ \\
& $-1.04 \pm \mathrm{j} 0.70$ & $-1.06 \pm \mathrm{j} 0.71$ & $-0.19 \pm \mathrm{j} 2.01$ & $-0.47 \pm \mathrm{j} 2.20$ \\
& & & $-0.32 \pm \mathrm{j} 3.02$ & $-0.68 \pm \mathrm{j} 3.21$ \\
& & & $-0.53 \pm \mathrm{j} 3.97$ & $-0.25 \pm \mathrm{j} 4.00$ \\
\hline
\end{tabular}

Table 4. Phase 1 controller gains

\begin{tabular}{|c|c|c|c|c|}
\hline $\begin{array}{l}\text { Pitch } \\
{\left[\mathrm{K}_{2}\right]^{T}}\end{array}$ & Units & \multicolumn{2}{|c|}{$\begin{array}{c}\text { Roll/Yaw* } \\
\qquad[\mathbf{K}]^{T}\end{array}$} & Units \\
\hline $4.2425 E+2$ & (ft-lb/rad) & $3.8526 \mathrm{E}+3$ & $3.7381 E+2$ & $(\mathrm{ft}-\mathrm{lb} / \mathrm{rad})$ \\
\hline $2.5412 E+5$ & (ft-lb-sec/rad) & $1.2003 \mathrm{E}+6$ & $1.0126 \mathrm{E}+5$ & (ft-lb-sec/rad) \\
\hline $1.4840 \mathrm{E}-2$ & (ft-lb/ft-lb-sec) & $1.4360 \mathrm{E}-2$ & $1.9364 \mathrm{E}-3$ & $(\mathrm{ft}-\mathrm{lb} / \mathrm{ft}-\mathrm{lb}-\mathrm{sec})$ \\
\hline $4.0150 \mathrm{E}-6$ & $\left(\mathrm{ft}-\mathrm{lb} / \mathrm{ft}-\mathrm{lb}-\mathrm{sec}^{2}\right)$ & $-1.6361 \mathrm{E}-6$ & $2.7852 \mathrm{E}-7$ & $\left(\mathrm{ft}-\mathrm{lb} / \mathrm{ft}-\mathrm{lb}-\mathrm{sec}^{2}\right)$ \\
\hline$-1.9064 \mathrm{E}-9$ & $\left(\mathrm{ft}-\mathrm{lb}-\mathrm{rad}^{2} / \mathrm{ft}-\mathrm{lb}-\mathrm{sec}^{3}\right)$ & $3.6578 \mathrm{E}-10$ & $-1.8526 \mathrm{E}-10$ & $\left(\mathrm{ft}-\mathrm{lb}-\mathrm{rad}^{2} / \mathrm{ft}-1 \mathrm{~b}-\mathrm{sec}^{3}\right)$ \\
\hline $2.1970 \mathrm{E}-6$ & $\left(\mathrm{ft}-\mathrm{lb}-\mathrm{rad}^{2} / \mathrm{ft}-\mathrm{lb}-\mathrm{sec}^{2}\right)$ & $7.6282 \mathrm{E}-7$ & $1.1857 \mathrm{E}-7$ & $\left(\mathrm{ft}-\mathrm{lb}-\mathrm{rad}^{2} / \mathrm{ft}-1 \mathrm{~b}-\mathrm{sec}^{2}\right)$ \\
\hline$-4.6097 \mathrm{E}-9$ & (ft-lb-rad $/$ ft-lb-sec $\left.{ }^{3}\right)$ & $-3.2712 \mathrm{E}-9$ & $-5.7517 \mathrm{E}-10$ & $\left(\mathrm{ft}-\mathrm{lb}-\mathrm{rad}^{2} / \mathrm{ft}-\mathrm{lb}-\mathrm{sec}^{3}\right)$ \\
\hline$-5.2383 E-7$ & (ft-lb-rad $\left./ \mathrm{ft}-1 \mathrm{~b}-\mathrm{sec}^{2}\right)$ & $-3.3865 E-7$ & $-1.6409 \mathrm{E}-7$ & $\left(\mathrm{ft}-\mathrm{lb}-\mathrm{rad}^{2} / \mathrm{ft}-\mathrm{lb}-\mathrm{sec}^{2}\right)$ \\
\hline$-5.3793 \mathrm{E}-9$ & (ft-lb-rad $\left./ \mathrm{ft}-\mathrm{lb}-\mathrm{sec}^{3}\right)$ & $-1.0702 \mathrm{E}-8$ & $-1.1317 \mathrm{E}-9$ & $\left(\mathrm{ft}-\mathrm{lb}-\mathrm{rad}^{2} / \mathrm{ft}-\mathrm{lb}-\mathrm{sec}^{3}\right)$ \\
\hline$-1.9423 \mathrm{E}-6$ & (ft-lb-rad $/$ ft-lb-sec $\left.{ }^{2}\right)$ & $-3.4827 \mathrm{E}-6$ & $-5.3664 \mathrm{E}-7$ & $\left(\mathrm{ft}-\mathrm{lb}-\mathrm{rad}^{2} / \mathrm{ft}-\mathrm{lb}-\mathrm{sec}^{2}\right)$ \\
\hline$-7.3458 \mathrm{E}-9$ & $\left(\mathrm{ft}-\mathrm{lb}-\mathrm{rad}^{2} / \mathrm{ft}-\mathrm{lb}-\mathrm{sec}^{3}\right)$ & $-1.5903 \mathrm{E}-8$ & $-1.5491 \mathrm{E}-9$ & $\left(\mathrm{ft} \cdot \mathrm{lb}-\mathrm{rad}^{2} / \mathrm{ft}-\mathrm{lb}-\mathrm{sec}^{3}\right)$ \\
\hline$-2.6056 \mathrm{E}-6$ & $\left(\mathrm{ft}-\mathrm{lb}-\mathrm{rad}^{2} / \mathrm{ft}-\mathrm{lb}-\mathrm{sec}^{2}\right)$ & $-3.1256 \mathrm{E}-6$ & $-4.7197 \mathrm{E}-7$ & $\left(\mathrm{ft}-\mathrm{lb}-\mathrm{rad}^{2} / \mathrm{ft}-\mathrm{lb}-\mathrm{sec}^{2}\right)$ \\
\hline & & $9.4016 \mathrm{E}+2$ & $2.4994 \mathrm{E}+2$ & $(\mathrm{ft}-\mathrm{lb} / \mathrm{rad})$ \\
\hline & & $-1.2743 E+5$ & $1.1386 \mathrm{E}+5$ & $(\mathrm{ft}-\mathrm{lb}-\mathrm{sec} / \mathrm{rad})$ \\
\hline & & $-2.4992 E-3$ & $-3.5209 \mathrm{E}-3$ & $(\mathrm{ft}-\mathrm{lb} / \mathrm{ft}-\mathrm{lb}-\mathrm{sec})$ \\
\hline & & $-7.3398 \mathrm{E}-7$ & $-1.0348 \mathrm{E}-6$ & $\left(\mathrm{ft}-\mathrm{lb} / \mathrm{ft}-\mathrm{lb}-\mathrm{sec}^{2}\right)$ \\
\hline & & $4.8557 \mathrm{E}-9$ & $-5.5935 \mathrm{E}-10$ & $\left(\mathrm{ft}-\mathrm{lb}-\mathrm{rad}^{2} / \mathrm{ft}-\mathrm{lb}-\mathrm{sec}^{3}\right)$ \\
\hline & & $3.7017 \mathrm{E}-7$ & $-4.2651 \mathrm{E}-6$ & $\left(\mathrm{ft}-\mathrm{lb}-\mathrm{rad}^{2} / \mathrm{ft}-\mathrm{lb}-\mathrm{sec}^{2}\right)$ \\
\hline & & $2.0608 \mathrm{E}-9$ & $-6.8224 \mathrm{E}-10$ & $\left(\mathrm{ft}-\mathrm{lb}-\mathrm{rad}^{2} / \mathrm{ft}-\mathrm{lb}-\mathrm{sec}^{3}\right)$ \\
\hline & & $1.8854 \mathrm{E}-6$ & $-2.4769 \mathrm{E}-6$ & $\left(\mathrm{ft}-\mathrm{lb}-\mathrm{rad}^{2} / \mathrm{ft}-\mathrm{lb}-\mathrm{sec}^{2}\right)$ \\
\hline & & $4.0142 \mathrm{E}-10$ & $9.4962 \mathrm{E}-10$ & $\left(\mathrm{ft}-\mathrm{lb}-\mathrm{rad}^{2} / \mathrm{ft}-\mathrm{lb}-\mathrm{sec}^{3}\right)$ \\
\hline & & $1.5548 \mathrm{E}-6$ & $-2.7820 \mathrm{E}-6$ & $\left(\mathrm{ft}-\mathrm{lb}-\mathrm{rad}^{2} / \mathrm{ft}-\mathrm{lb}-\mathrm{sec}^{2}\right)$ \\
\hline & & $8.3363 \mathrm{E}-10$ & $8.3453 \mathrm{E}-10$ & $\left(\mathrm{ft}-\mathrm{lb}-\mathrm{rad}^{2} / \mathrm{ft}-\mathrm{lb}-\mathrm{sec}^{3}\right)$ \\
\hline & & $1.3125 \mathrm{E}-6$ & $-2.5757 \mathrm{E}-6$ & $\left(\mathrm{ft}-\mathrm{lb}-\mathrm{rad}^{2} / \mathrm{ft}-\mathrm{lb}-\mathrm{sec}^{2}\right)$ \\
\hline
\end{tabular}

* Designed for a pitch TEA of $0^{\circ}$ 

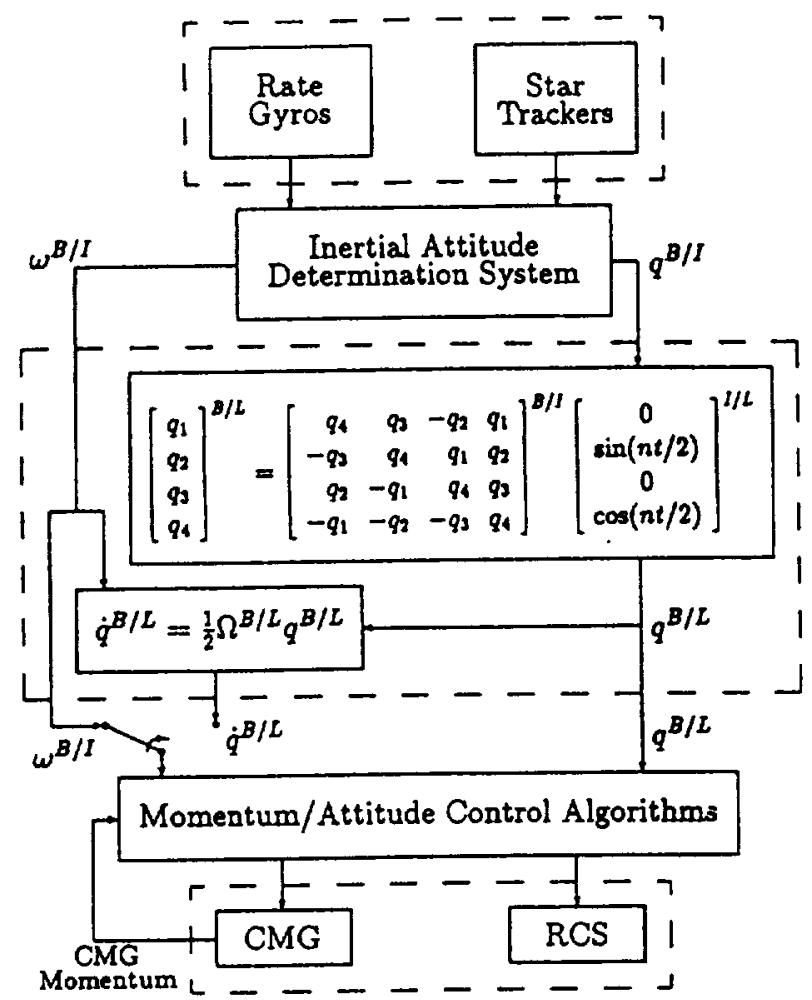

$B / I$ indicates body frame with respect to inertial frame $B / L$ indicates body frame with respect to LVLH frame $\mathrm{I} / \mathrm{L}$ indicates inertial frame with respect to LVLH frame

Fig. 1. A quaternion feedback control system for the Space Station.

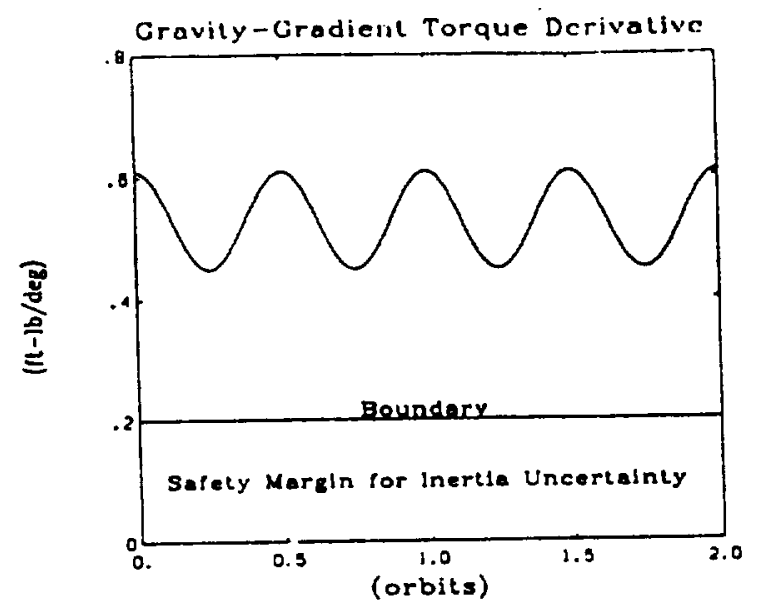

Fig. 2. Time-varying pitch-axis gravity-gradient torque derivative.

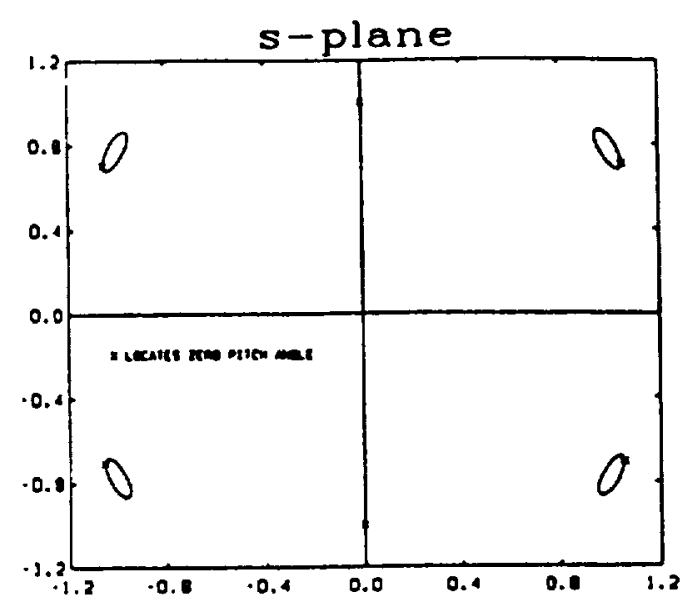

Fig. 3. Plot of roll/yaw open-loop poles versus pitch angle in the s plane ( $s$ in units of orbital rate).
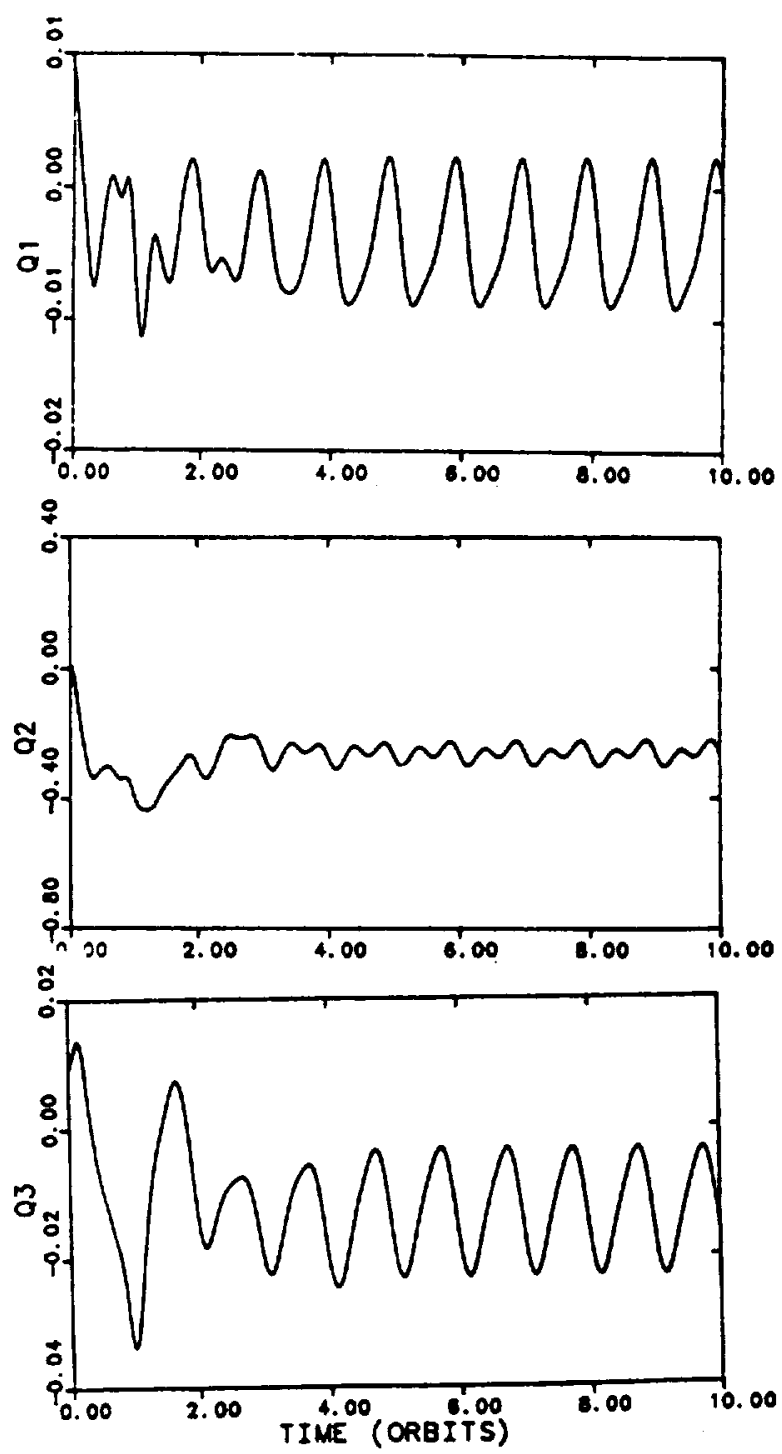

Fig. 4. Closed-loop quaternion responses. 

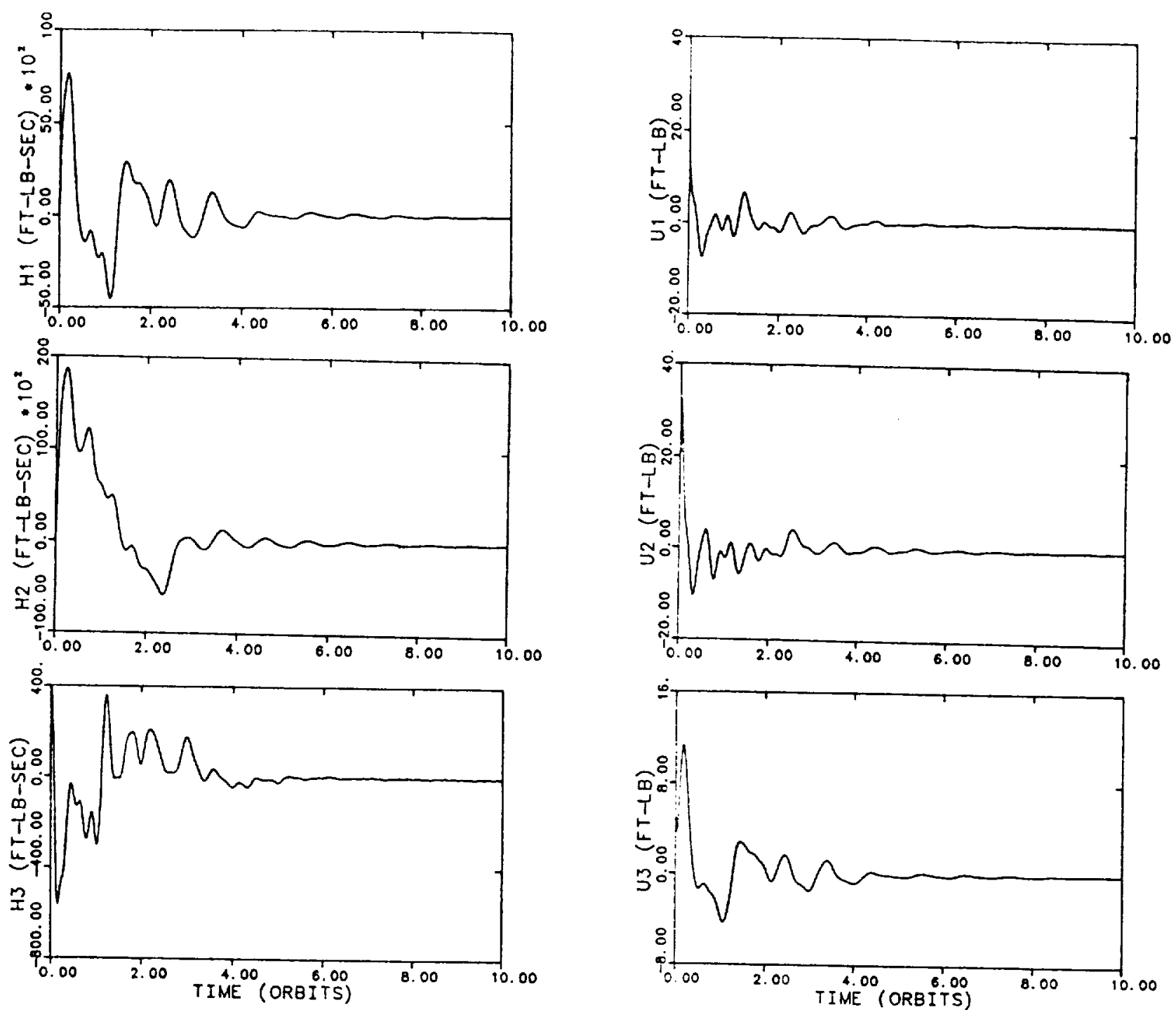

Fig. 5. CMG momentum histories.

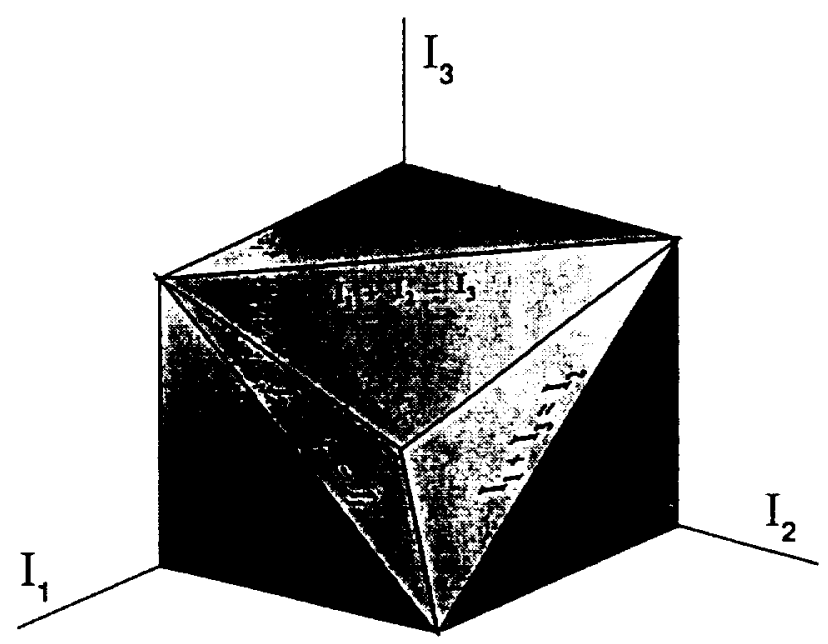

Fig. 6. Commanded torque histories.

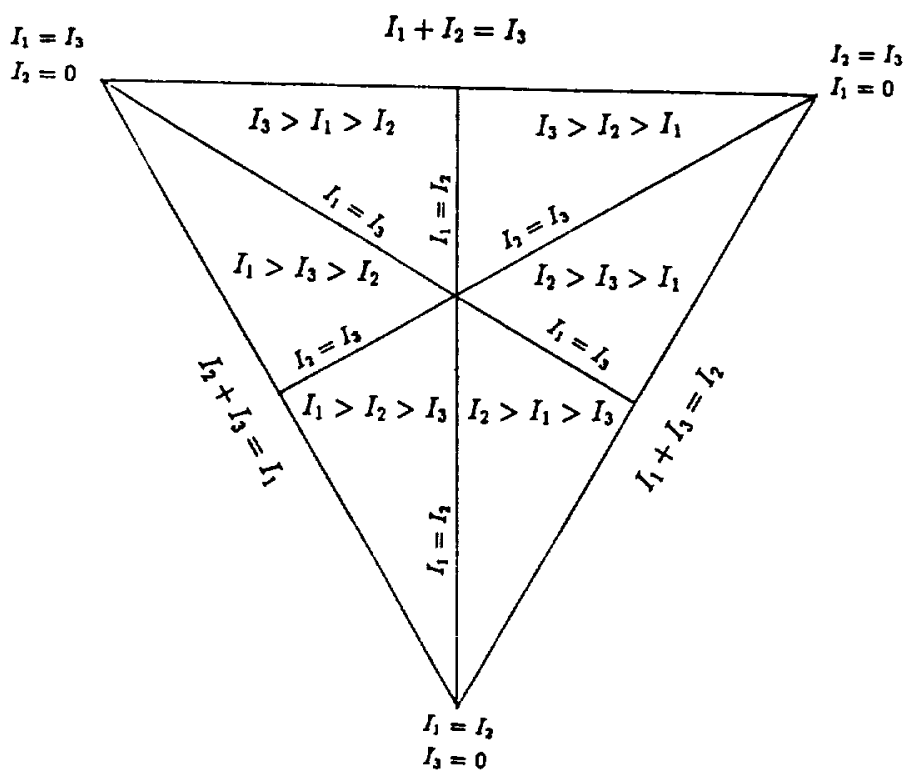

Fig. A.1. Physical boundaries of inertia values.

Fig. A.2. Regions within the physical boundaries. 